\title{
IdeAs
}

Idées d'Amériques

$6 \mid 2015$

Migrer dans les Amériques

\section{Gildas Simon (Dir.), Dictionnaire des migrations internationales, approche géohistorique}

Paris, Armand Colin, 2015, 807 p.

Josepha MILAZZO

\section{OpenEdition}

\section{Journals}

Édition électronique

URL : https://journals.openedition.org/ideas/1234

DOI : 10.4000/ideas.1234

ISSN : 1950-5701

Éditeur

Institut des Amériques

Référence électronique

Josepha MILAZZO, « Gildas Simon (Dir.), Dictionnaire des migrations internationales, approche géohistorique », IdeAs [En ligne], 6 | 2015, mis en ligne le 16 décembre 2015, consulté le 19 octobre 2022. URL : http://journals.openedition.org/ideas/1234; DOI : https://doi.org/10.4000/ideas.1234

Ce document a été généré automatiquement le 19 octobre 2022.

\section{c) $(1)$}

Creative Commons - Attribution - Pas d'Utilisation Commerciale - Pas de Modification 4.0 International - CC BY-NC-ND 4.0

https://creativecommons.org/licenses/by-nc-nd/4.0/ 


\section{Gildas Simon (Dir.), Dictionnaire des migrations internationales, approche géohistorique}

Paris, Armand Colin, 2015, 807 p.

Josepha MILAZZO

\section{RÉFÉRENCE}

Gildas Simon (Dir.), Dictionnaire des migrations internationales, approche géohistorique, Paris, Armand Colin, 2015, $807 \mathrm{p}$.

1 Le Dictionnaire des migrations internationales, publié en 2015 aux Éds. Armand Colin, sous la direction de Gildas Simon, -géographe, ancien Professeur à l'Université de Poitiers, fondateur du Laboratoire Migrinter et de la Revue Européenne des Migrations Internationales -, propose un état des lieux de l'espace migratoire mondial selon une lecture des États du monde au prisme de leur histoire migratoire individuelle, depuis le $\mathrm{XVI}^{\mathrm{ème}}$ siècle jusqu'à nos jours. Il s'agit d'une encyclopédie étatique de l'histoire des mouvements internationaux de populations : cette compilation imposante de plus de 800 pages inventorie 190 des 208 États recensés au monde (Nations Unies, 2015). Elle vise à constituer, pour le plus grand nombre, un outil de compréhension des « raisons profondes » de la migration, de sorte à ce que le public puisse se forger une opinion propre et appréhender au mieux ce sujet d'actualité, au cœur de la vie socio-politique et aux prises de nombre d' "amalgames » et d' "instrumentalisations » (Simon G., $2015: 11)$.

2 Le choix du prisme géopolitique de l'État-Nation comme espace de référence pour l'observation des flux migratoires internationaux s'explique, dans le contexte de la mondialisation, par le fait que son action demeure déterminante dans leur façonnement: l'introduction suggère en effet que la migration internationale, enjeu majeur des politiques nationales et supranationales, place au cœur des relations 
interétatiques la question de sa gestion; au point qu'analyser les rapports d'un État à la question migratoire est un moyen d'interroger les fondements de celui-ci. Sur la forme, l'entrée par l'État constitue par ailleurs une modalité efficace de synthétisation et de présentation d'une telle masse d'informations : le format 'notice' rend la comparaison État-État possible, aux échelles intra et interrégionales, ceci entre les espaces, les époques et les logiques migratoires explicitées (immigration/émigration); ce qui est propice à la compréhension de la diversité des configurations étatiques migratoires dans le monde. Les pays-notices sont classées par ordre alphabétique à l'intérieur de quinze sous-continents/aires culturelles et sociopolitiques, identifiés comme autant d'ensembles migratoires régionaux révélateurs de l'organisation économique mondiale, faisant office de sous-parties de l'ouvrage, elles-mêmes rassemblées sous neuf continents et ensembles géopolitiques. Ces derniers sont introduits par un bref propos qui situe les caractéristiques migratoires de ces ensembles suprarégionaux au sein du système mondial, et par une carte politique qui localise les États dans l'aire géographique donnée. Chaque notice débute par la singularité du paysage migratoire du pays, synthétiquement contextualisée à l'aide de données statistiques émanant d'institutions nationales et internationales recensées en fin d'ouvrage. Un développement retrace ensuite, selon les époques successives, les tendances immigration/émigration du pays, dans une démarche géohistorique, depuis les Grandes Découvertes, -époque d'intensification des échanges et des mouvements humains, aube de la « première » mondialisation-, jusqu'à ce jour.

3 L'ouvrage souligne d'une part l'importance de la participation de la migration à la reproduction sociale, au titre de phénomène indissociable du développement des sociétés. D'autre part, l'approche historique, illustrant que tout pays a été à une époque caractérisé plutôt par l'émigration ou l'immigration, permet de (re)mettre sur un même pied d'égalité l'ensemble des États : cette démarche s'éloigne ainsi de visions statiques pouvant enfermer un pays dans des logiques de rapports de domination, caractérisé qu'il puisse être comme " pays d'émigration » ou « d'immigration ».

Outre l'état de la connaissance, la longueur de chaque notice dépend aussi du poids démographique et de l'importance historique et actuelle des dynamiques migratoires du pays traité. Ceci explique le léger différentiel dans l'effectif de pays présentés (190 vs 208), outre le regroupement d'États constitutifs de systèmes migratoires telle la "Caraïbe orientale ». Les États archipélagiques de l'Océanie qui compte ici l'Australie, la République de Fidji et la Nouvelle-Zélande, sont quelque peu absents; un fait qui questionne le manque possible de chercheurs sur ces espaces, le défaut éventuel de connaissances à leur sujet, "trous noirs de la connaissance» (p.11), et surtout qui suscite la curiosité quant à la nature des mouvements humains en ces contrées. Un système de renvoi aux pays ayant (eu) un lien migratoire avec celui traité conclut chaque notice.

5 L'éditeur propose aussi des ressources en ligne gratuites, dont l'ouvrage papier est en partie exempt : la bibliographie complète ordonnée par pays (environ 1500 titres) et des articles de référence. L'ouvrage est synthétique, didactique, sa force réside dans le fait qu'il soit accessible à tous. Il s'agit de l'aboutissement d'un projet collectif de vaste envergure qui repose sur la contribution d'une équipe internationale et multidisciplinaire en sciences humaines et sociales d'environ 150 contributeurs, chercheurs expérimentés comme jeunes, spécialistes des pays traités sinon d'aires géographiques englobant espaces de provenance et/ou d'arrivée de populations 
migrantes étudiées; les nombreuses disciplines représentées témoignent de l'étendue de l'intérêt scientifique, de la diversité des approches, et des enjeux d'un dialogue transdisciplinaire sur la migration, autant d'arguments à la réalisation de ce dictionnaire. À ce titre, plusieurs notions et concepts-clefs apparaissent au fil du texte, dont une définition est donnée en début d'ouvrage : les thèmes associés « dont l'étude procède d'autres approches que celles adoptées ici » (Simon G., 2015 : 13) suggèrent qu'un ouvrage épistémologique et transdisciplinaire sur la migration reste à construire et pourrait faire l'apanage d'une prochaine publication, ce dans la veine de ce dictionnaire qui vient grandement enrichir la production éditoriale de l'époque sur les migrations et qui a vocation à avoir une portée internationale dans les études migratoires.

\section{AUTEURS}

\section{JOSEPHA MILAZZO}

Josepha Milazzo est géographe, doctorante en cotutelle internationale de thèse à l'Université d'Aix-Marseille (TELEMMe) et à l'Université Autonome de Barcelone (Département de géographie), sous les directions de Virginie Baby-Collin et de Ricard Morén-Alegret. Ses recherches doctorales ont reçu les soutiens financiers de l'Institut des Amériques, du Social Science Research Council et des Universités d'Aix-Marseille et Autonome de Barcelone. Ses travaux interrogent jusqu'alors la participation des migrants internationaux aux transformations de petites localités périphériques d'arrivée et/ou d'immigration dans les contextes de pays aux revenus élevés de l'Europe méditerranéenne (Espagne, Catalogne et France, Corse), ceci au prisme de changements agricoles et touristiques dans le contexte de la mondialisation. Elle est membre du pôle événementiel du Réseau Migrations depuis Juin 2014 à ce jour.

jjomilazzo@gmail.com / josepha.milazzo@yahoo.fr 